\title{
Variabilidade interensaios de dislipidemias em pacientes hipertensos
}

\author{
Inter-assays variability of dislipidemias in hypertensive patients
}

Nilton Rosini'; Andreza Diegoli Rosini²; Guilherme Diegoli Rosini³; Celso Spada4; Arício Treitinger ${ }^{4}$; Donaida Maria Mousse ${ }^{5}$

\begin{tabular}{l}
\multicolumn{1}{c|}{ Unitermos } \\
Não-HDL-C \\
LDL-C \\
Pacientes hipertensos \\
Variabilidade interensaios
\end{tabular}

Variabilidade interensaios

\section{resumo}

Introdução e objetivos: Ensaios de diferentes procedências para avaliação das dislipidemia podem resultar em variações significativas nos resultados obtidos e consequente conduta inadequada pelo clínico. O estudo objetivou comparar resultados laboratoriais de colesterol total (CT), triglicérides (TG), colesterol da lipoproteína de alta densidade (HDL-C), colesterol da lipoproteína de baixa densidade (LDL-C), apolipoproteína A-1 (Apo A-1), apolipoproteína B (Apo B) e lipoproteína (a) (Lp[a]) e índices lipídicos (não-HDL-C, CT/HDL-C, LDL-C/HDL-C, TG/HDL-C e Apo B/HDL-C) de pacientes hipertensos e/ou diabéticos diagnosticados. Métodos: Foram utilizados conjuntos reativos, e os respectivos analisadores Gold Analisa, Dia Sys (CCX - Abbott), Dade Behring (Nefelômetro BN 100) e Roche (COBAS Integra 400), para verificar a reprodutibilidade dos resultados obtidos. Participaram 99 pacientes ( 36 do sexo masculino e 63 do feminino). Comparando os resultados, verificamos que: todas as médias obtidas dos constituintes lipídicos apresentaram diferença significativa; número semelhante de pacientes apresentou níveis séricos elevados de $C T, T G, L p(a)$ e Apo A-1. O HDL-C, o LDL-C e a Apo B apresentaram discordância, assim como os índices de CT/LDL-C, LDL-C/HDL-C e TG/HDL-C. Para não-HDL-C e ApoB/HDL, houve semelhança no número de pacientes com valores não recomendados. Em consequência da diferença, em relação ao LDL-C, a decisão da conduta terapêutica poderá ser inadequada, enquanto o não-HDL-C, além de evidenciar partículas aterogênicas, apresentou número de hipertensos com valores séricos não referendados semelhantes, independente da metodologia e do equipamento utilizado. Conclusão: No grupo de hipertensos analisados, o não-HDL-C se caracterizou um importante fator de correção interensaios de parâmetros lipídicos. E sua associação à relação Apo B/HDL-C pode ser um fator adicional em relação às condutas hipolipemiantes a serem adotadas.

abstract

Introduction and objectives: Different assays to evaluate dyslipidemia may show significant variations in the obtained results and a consequent inappropriate clinical approach may be adopted. This study aimed to compare the results of total cholesterol (CT), triglycerides (TG), HDL-C, Apo A1, Apo B, lipoprotein (a) and lipidic indexes (not-HDL-C, CT/HDL-C, LDL-C/HDL-C, TG/HDL-C and Apo B/HDL-C) of hypertensive and/or diabetic patients. Methods: The following reactive kits and respective analyzers were applied to verify the reproducibility of results: Gold Analisa, DiaSys (CCX-ABBOTT), Dade Behring (Nephelometer BN 100) and Roche (COBAS Integra 400). Ninety nine patients (36 male and 63 female gender) were investigated. Comparing the results, we observed that all mean numbers of lipid constituents showed a significant difference. A similar number of patients had high $C T, T G, L p(a)$ and Apo A-1 serum levels. There was also disagreement in HDL-C, LDL-C, ApoB, CT/LDL-C, LDLC/HDL-C and TG/HDL-C indexes. For not-HDL-C and ApoB/HDL, there was similarity in the number of patients with not recommended values. As a consequence of this difference, the choice of therapeutic approach may be inappropriate as to LDL-C levels, whereas Not-HDL-C not only showed atherogenic particles but also a number of hypertensive patients with similar not recommended serum values, regardless of the methodology and the equipment used. Conclusion: In the analyzed group of hypertensive patients, not-HDL-C was an important inter assay correction factor of lipidic parameters. The association with Apo B/HDL-C relation may be an additional factor as to the choice of hypolipemiant treatments.

\section{key words}

Not-HDL-C

LDL-C

Hypertensive patients

Inter-assays variability

1. Mestre; farmacêutico bioquímico.

2. Farmacêutica bioquímica.

3. Biólogo.

4. Professor-doutor.

5. Mestra; farmacêutica bioquímica. 


\section{Introdução}

As doenças cardiovasculares (DCVs) são responsáveis por mais de 30\% dos desfechos clínicos fatais no Brasil, destacando-se principalmente o acidente vascular cerebral $(A V C)$ e a doença arterial coronariana (DAC) ${ }^{(6)}$.

A associação da doença aterosclerótica à dislipidemia é relatada desde a década de 30 do século passado ${ }^{(24)} \mathrm{e}$ vários estudos têm corroborado esta teoria ${ }^{(7,12,14)}$. Mais recentemente o estudo INTERHEART comprovou de forma incontestável esta associação por meio de pesquisas realizadas em 52 países ${ }^{(40)}$. A dislipidemia é frequente em hipertensos ${ }^{(32)}$ e pode levar à hipertensão ${ }^{(13)}$. Nos pacientes de alto risco para DCV, a redução dos níveis séricos de lipídios, principalmente lipoproteína de baixa densidade (LDL), pode resultar em maior benefício em relação à prevenção da DAC(11). Contudo, para definir a meta adequada a ser alcançada e a intensidade da abordagem terapêutica, são necessárias a avaliação e a estratificação individuais dos riscos com maior precisão, exatidão e padronização dos métodos empregados na sua determinação(39).

Para avaliação laboratorial das dislipidemias se encontram disponíveis ensaios manuais e automatizados de diferentes procedências, cujos princípios metodológicos não são variados, podendo, no entanto, resultar em variações significativas dos resultados obtidos. Assim, avaliações equivocadas dos resultados laboratoriais dos níveis séricos dos diferentes lipídios podem ocorrer, originando erros na interpretação e na adequação da conduta terapêutica pelo clínico em relação aos valores referendados pelas diretrizes. Além disso, as possíveis variações laboratoriais não estariam necessariamente sendo consideradas.

Levando-se em conta o envolvimento das dislipidemias na gênese da aterosclerose, o estudo objetivou comparar os resultados laboratoriais dos principais constituintes lipídicos: colesterol total (CT), triglicérides (TG), colesterol da lipoproteína de alta densidade (HDL-C), colesterol da lipoproteína de baixa densidade (LDL-C), apolipoproteína A-1 (Apo A-1), apolipoproteína $B(A p o B)$, lipoproteína (a) (Lp [a]) e índices lipídicos (não-HDL-C, CT/HDL-C, LDL-C/HDL-C, TG/HDL-C e Apo B/HDL-C) em pacientes hipertensos e/ou diabéticos diagnosticados, por meio de ensaios de diferentes procedências realizados em três analisadores automatizados (CCX - ABBOTT, Nefelometria BN 100, DADE BEHRING e COBAS Integra 400 - ROCHE) para verificar a reprodutibilidade dos resultados obtidos.

\section{Materiais e métodos}

Participaram deste estudo 99 pacientes com diagnóstico de hipertensão submetidos à terapia medicamentosa prescrita por profissional médico habilitado, sendo que, destes, 20 eram, além de hipertensos, também diabéticos diagnosticados. Dos pacientes que participaram deste estudo, 36 eram do sexo masculino e 63 do feminino. As amostras de sangue foram colhidas após jejum de 12 a 14 horas e realizadas as determinações dos níveis séricos de CT, TG, HDL-C, LDL-C, Apo A-1, Apo B e Lp(a). Os constituintes lipídicos anteriormente descritos foram quantificados em equipamentos CCX (Abbott), Nefelômetro BN 100 (Dade Behring) e COBAS-Integra 400 (Roche) utilizando conjuntos reativos conforme demonstrado na Tabela 1. As metodologias analíticas utilizadas no estudo encontram-se na Tabela 2. Os níveis de não-HDL-C foram calculados utilizando-se a equação da fórmula: não-HDL-C = CT - HDL-C. As relações CT/HDL-C, LDL-C/HDL-C, TG/HDLC e Apo B/HDL-C foram obtidas a partir dos níveis séricos destes constituintes. As determinações dos níveis séricos dos constituintes lipídicos avaliados foram realizadas no Laboratório Verner Willrich, em Brusque, Santa Catarina. Todos os pacientes que consentiram em participar do estudo assinaram o termo de consentimento livre e esclarecido. O presente estudo é parte integrante do Estudo HDB, que foi aprovado pelo Comitê de Ética de Pesquisa com Seres Humanos na Universidade Federal de Santa Catarina (UFSC), Parecer no 383/05.

Todos os parâmetros lipídicos apresentam o mesmo princípio analítico, divergindo em relação às concentrações dos insumos, exceção feita ao HDL-C, em que o DiaSys emprega anticorpo anti- $\beta$-lipoproteína humana (anti-LDL, lipoproteína de muito baixa densidade [VLDL] e quilomícrons), enquanto o COBAS Integra utiliza enzimas modificadas por polietilenoglicol (PEG) na presença de sulfato de magnésio e sulfato de dextrano.

Os parâmetros lipídicos, seguindo orientação dos procedimentos técnicos dos fabricantes, foram aferidos com os seguintes calibradores: CCX (Trucal - DiaSys); Nefelômetro (N Apolipoprotein Standard Serum e N Lp(a) Standard SY) e COBAS Integra (CFAS e CFAS lipid). Para a garantia de qualidade das determinações foram utilizados os seguintes controles: CCX (TruLab N); nefelômetro (Apolipoprotein CHD e N Lp(a) Contro SY) e COBAS Integra (Precinorm, Precinorm lipides e Control Set Lipoprot [a]), de acordo com o ensaio e o respectivo equipamento. 
Equipamento, conjunto reativo e procedência dos reagentes para determinação dos

Tabela 1 constituintes lipídicos estudados

\begin{tabular}{|c|c|c|c|}
\hline \multirow{2}{*}{ Constituinte Lipídico } & \multicolumn{3}{|c|}{ Equipamento/Conjunto reativo/Procedência } \\
\hline & $\operatorname{ccx}$ & Nefelômetro BN 100 & COBAS-Integra 400 \\
\hline Colesterol Total & $\begin{array}{l}\text { Colesterol-PP } \\
\text { (Gold Analisa) }\end{array}$ & - & $\begin{array}{l}\text { Cholesterol Gen.2 } \\
\text { (Roche) }\end{array}$ \\
\hline Triglicérides & Triglycerides FS (DiaSys) & - & $\begin{array}{l}\text { Triglycerides } \\
\text { (Roche) }\end{array}$ \\
\hline HDL-C & HDL-C Imuno FS (DiaSys) & - & $\begin{array}{l}\text { HDL-Cholesterol plus 2nd } \\
\text { generation } \\
\text { (Roche) }\end{array}$ \\
\hline LDL-C & LDL-C Select FS (DiaSys) & - & $\begin{array}{l}\text { LDL-Cholesterol plus 2nd } \\
\text { generation } \\
\text { (Roche) }\end{array}$ \\
\hline Apo A-1 & - & $\begin{array}{l}\text { N Antisera to Human } \\
\text { Apolipoprotein A-I } \\
\text { (Dade Behring) }\end{array}$ & $\begin{array}{c}\text { Tina-quant Apolipoprotein A-1 } \\
\text { ver.2 } \\
\text { (Roche) }\end{array}$ \\
\hline Apo B & - & $\begin{array}{l}\text { N Antisera to Human } \\
\text { Apolipoprotein B } \\
\text { (Dade Behring) }\end{array}$ & $\begin{array}{c}\text { Tina-quant Apolipoproein B } \\
\text { ver.2 } \\
\text { (Roche) }\end{array}$ \\
\hline Lp(a) & - & $\begin{array}{l}\text { N Latex Lp(a) Reagent } \\
\text { (Dade Behring) }\end{array}$ & $\begin{array}{l}\text { Tina-quant Lipoprotein(a) (Látex) } \\
\text { (Roche) }\end{array}$ \\
\hline
\end{tabular}

Tabela 2

Constituinte Lipídico

Colesterol Total

Triglicérides

HDL-C

LDL-C

Apo A-1

Apo B

$L p(a)$
Constituintes lipídicos estudados nos diferentes equipamentos e metodologias analíticas utilizadas neste estudo

Enzimático colorimétrico
Enzimático colorimétrico
Imuno enzimático colorimétrico
Enzimático colorimétrico
homogêneo direto

Imunoturbidimétrico

Imunoturbidimétrico

Imunoturbidimétrico
COBAS Integra 400

Enzimático colorimétrico

Enzimático colorimétrico

Enzimático colorimétrico homogêneo

Enzimático colorimétrico homogêneo direto Imunoturbidimétrico Imunoturbidimétrico Imunoturbidimétrico
A variabilidade analítica (coeficiente de variação analítica) dos equipamentos foi determinada pelo ensaio de repetição da mesma amostra (soro de paciente participante do estudo) em 10 corridas independentes nas mesmas condições experimentais (determinações sequenciais). As médias, o desvio padrão e o coeficiente de variação obtidos estão demonstrados na Tabela 3.
A comparação dos níveis séricos dos constituintes lipídicos estudados, bem como as relações entre estes, foi realizada pelo teste $t$ de Student para amostras dependentes, utilizando o programa estatístico Instat 2. As diferenças entre as médias obtidas, pela determinação dos mesmos parâmetros lipídicos, dos mesmos pacientes, em equipamentos diferentes, foram consideradas significativas quando $p<0,05$. 


\section{Tabela 3}

Médias, desvio padrão e coeficiente de variação da amostra, para verificar o coeficiente de

Constituinte Lipídico variação analítica

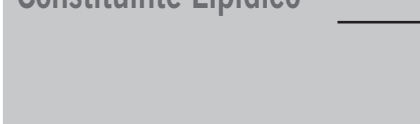

Colesterol Total(mg/dl)

$\begin{array}{cc}\text { CCX } & \text { Nefelômetro BN100 } \\ \text { Média(mg/dL), } & \text { Média(mg/dL), } \\ \text { DP(mg/dL, CV(\%) } & \text { DP(mg/dL, CV(\%) }\end{array}$

Triglicérides(mg/dl)

$196,8 \pm 1,77 ; 0,89$

$\mathrm{HDL}-\mathrm{c}(\mathrm{mg} / \mathrm{dl})$

$139,7 \pm 3,41 ; 2,44$

$55 \pm 1,48 ; 2,69$

LDL-c(mg/dl)

$97,9 \pm 1,92 ; 1,96$

Apo A-1 (mg/dl)

$128,9 \pm 1,1 ; 0,85$

$106,9 \pm 1,3 ; 1,21$

COBAS Integra 400

Apo B(mg/d)

$27,8 \pm 0,5 ; 1,8$

Média(mg/dL),

DP(mg/dL, CV(\%)

$\mathrm{Lp}(\mathrm{a})(\mathrm{mg} / \mathrm{dl})$

$212,7 \pm 1,26 ; 0,59$

$151,7 \pm 2,73 ; 1,79$

$51,1 \pm 0,93 ; 1,82$

$106,4 \pm 1,81 ; 1,70$

$134,7 \pm 1,65 ; 1,22$

$112,5 \pm 1,4 ; 1,24$

$\mathrm{DP}=$ desvião padrão; $\mathrm{CV}$ = coeficiente de variação.

\section{Resultados}

Os níveis séricos dos constituintes lipídicos $\mathrm{CT}$, TG, HDL-C e LDL-C determinados pelos equipamentos CCX e COBAS Integra 400, assim como os de Apo A-1, Apo B e $L p(a)$ determinados pelo nefelômetro BN100 e COBAS Integra 400 , apresentaram diferença significativa $(p<0,0001)$ nos pacientes estudados quando analisadas as médias obtidas (Tabela 4).

Quando analisamos a Tabela 5 verificamos que o número de pacientes que apresentaram níveis séricos de CT, TC e Lp (a) elevados era semelhante, apesar de terem sido utilizados reagentes de procedência diferente, assim como equipamentos distintos. Com relação aos níveis séricos de Apo A-1 observamos que eles se encontravam no limite de normalidade estabelecido. Entretanto, o número de pacientes que apresentaram níveis séricos de HDL-C, LDL-C e Apo B em desacordo com os valores de referência recomendados pelas III Diretrizes Brasileiras sobre Dislipidemias foi diferente.

As médias das relações CT/LDL-C, LDL-C/HDL-C, TG/HDLC e Apo B/HDL-C, assim como os níveis séricos de não-HDL-C, apresentaram diferenças significativas $(p<0,0001)$ quando comparamos os resultados obtidos a partir de determinações realizadas nos equipamentos $C C X /$ Nefelômetro com aqueles obtidos em equipamento COBAS Integra 400 (Tabela 6).

$\mathrm{O}$ número de pacientes que apresentaram valores de CT/LDL-C, LDL-C/HDL-C e TG/HDL-C em desacordo com os valores de referência recomendados foi discordante quando estes índices foram calculados a partir dos obtidos pelas diferentes metodologias e equipamentos utilizados. No entanto, observamos que o número de pacientes que apresentaram níveis de não-HDL-C e Apo B/HDL em desacordo com os valores de referência foi semelhante (Tabela 7).

\begin{tabular}{lcccc} 
& \multicolumn{4}{c}{$\begin{array}{l}\text { Níveis séricos de CT, TG, HDL-c, LDL-c, Apo A - 1, Apo B e Lp(a), em mg/dl obtidos dos } \\
\text { pacientes nos equipamentos estudados }\end{array}$} \\
\cline { 2 - 4 } Constituintes lipídicos 4 & CCX & Nefelômetro BN100 & COBAS Integra 400 & $r^{*}$ \\
\cline { 2 - 4 } & Média \pm DP & Média \pm DP & Média \pm DP & \\
Colesterol total & $226,3 \pm 42,0$ & - & $230,8 \pm 43,0^{* *}$ & 0,9356 \\
Triglicérides & $171,8 \pm 113,1$ & - & $189,8 \pm 124,7^{* *}$ & 0,9925 \\
HDL-c & $56,7 \pm 14,6$ & - & $48,7 \pm 13,8^{* *}$ & 0,9040 \\
LDL-C & $101,2 \pm 28,6$ & - & $145,2 \pm 37,1^{* *}$ & 0,8951 \\
Apo A-1 & - & $161,6 \pm 29,1$ & $168,4 \pm 33,6^{* *}$ & 0,7879 \\
Apo B & - & $113,9 \pm 29,3$ & $95,8 \pm 26,0^{* *}$ & 0,7075 \\
Lp(a) & - & $31,2 \pm 34,7$ & $25,8 \pm 26,2^{* *}$ & 0,8464
\end{tabular}

* Coeficiente de correlação. **Significância estatística $(p<0,0001)$. DP = Desvio Padrão. 
Tabela 5

Número de pacientes com níveis séricos dos constituintes lipídicos em desacordo com o recomendado pelas III Diretrizes Brasileiras sobre Dislipidemias, de acordo com 0 equipamento utilizado

\begin{tabular}{lccc}
\hline Constituinte Lipídico & CCX & Nefelômetro BN 100 & COBAS Intera 400 \\
CT : $200-230 \mathrm{mg} / \mathrm{dl}$ & 37 & - & 36 \\
CT $>230 \mathrm{mg} / \mathrm{dl}$ & 37 & - & 42 \\
Total CT > $200 \mathrm{mg} / \mathrm{dl}$ & 74 & - & 78 \\
HDL-c Homens * & 08 & - & 17 \\
HDL-c Mulheres* & 16 & - & 34 \\
Total HDL-c & 24 & - & 31 \\
LDL-c $100-130 \mathrm{mg} / \mathrm{dl}$ & 30 & - & 64 \\
LDL $>130 \mathrm{mg} / \mathrm{dl}$ & 18 & - & 95 \\
Total LDL-c>100mg/dl & 48 & $n e n h u m$ & 48 \\
TG $>150 \mathrm{mg} / \mathrm{dl}$ & 41 & 3 & $n e n h u m$ \\
Apo A & - & 12 & 1 \\
Apo B Homens ** & - & 15 & 8 \\
Apo B Mulheres** & - & 24 & 9 \\
Total Apo B & - & &
\end{tabular}

* HDL-c: Homens < $40 \mathrm{mg} / \mathrm{dl}$, Mulheres $<50 \mathrm{mg} / \mathrm{dl}$.

** Apo B: Homens > 140 mg/dl (Nefelômetro), > 133 mg/dl (COBAS Integra); Mulheres > $125 \mathrm{mg} / \mathrm{dl}$ (Nefelômetro), > $117 \mathrm{mg} / \mathrm{dl}$ (COBAS Integra).

Tabela 6

Relações CT/LDL-c, LDL-c/HDL-c, TG/HDL-c e ApoB/HDL e níveis séricos de não-HDL-c obtidas a partir das determinações realizadas em CCX/ Nefelômetro BN 100 e em COBAS

\begin{tabular}{lccc}
\hline \multirow{2}{*}{ Índices } & CCX/Nefelômetro & COBAS Integra 400 & $r^{* *}$ \\
\cline { 2 - 3 } Não-HDL-c * & Média \pm DP & Média \pm DP & \\
CT/LDL-c & $169,6 \pm 43,0$ & $182,1 \pm 45,1^{* * *}$ & 0,9514 \\
LDL-C/HDL-c & $2,31 \pm 0,37$ & $1,61 \pm 0,21^{* * *}$ & 0,5108 \\
TG/HDL-c & $1,93 \pm 0,75$ & $3,22 \pm 1,26^{* * *}$ & 0,9063 \\
Apo B/HDL-c & $3,46 \pm 3,11$ & $4,68 \pm 5,16^{* * *}$ & 0,9652 \\
& $2,26 \pm 0,84$ & $2,17 \pm 0,92^{* * *}$ & 0,7732
\end{tabular}

*Valores em mg/dl. ** Coeficiente de correlação. ** Significância estatística $(p<0,0001)$.

Todos os pacientes da relação Apo B/HDL-C superior ao limite recomendado apresentaram níveis séricos de não-HDL-C elevados, independente da metodologia analítica e dos equipamentos utilizados na determinação dos constituintes lipídicos. Em relação às médias dos níveis sé- ricos de não-HDL-C obtidas pelos diferentes equipamentos utilizados, verificamos que as dos pacientes com relação Apo $\mathrm{B} / \mathrm{HDL}-\mathrm{C}$ recomendada eram significativamente maiores do que as daqueles que apresentaram relação Apo B/HDL-C superior à recomendada (Tabela 8). 
Relações CT/LDL-c, LDL-c/HDL-c, TG/HDL-c e ApoB/HDL e níveis séricos de não-HDL-c obtidas a partir das determinações realizadas em CCX/ Nefelômetro BN 100 e em COBAS

Tabela 6 Integra 400

\begin{tabular}{lccc}
\hline \multirow{2}{*}{ Índices } & CCX/Nefelômetro & COBAS Integra 400 & $r^{* *}$ \\
\cline { 2 - 3 } Não-HDL-c * & Média \pm DP & Média \pm DP & \\
CT/LDL-c & $169,6 \pm 43,0$ & $182,1 \pm 45,1^{* * *}$ & 0,9514 \\
LDL-c/HDL-c & $2,31 \pm 0,37$ & $1,61 \pm 0,21^{* * *}$ & 0,5108 \\
TG/HDL-c & $1,93 \pm 0,75$ & $3,22 \pm 1,26^{* * *}$ & 0,9063 \\
Apo B/HDL-c & $3,46 \pm 3,11$ & $4,68 \pm 5,16^{* * *}$ & 0,9652 \\
Nann & $2,26 \pm 0,84$ & $2,17 \pm 0,92^{* * *}$ & 0,7732
\end{tabular}

*Valores em mg/dl. ** Coeficiente de correlação. *** Significância estatística $(p<0,0001)$.

Tabela 7

Número de pacientes com índices lipídicos superiores ao limite desejado, de acordo com as

\begin{tabular}{|c|c|c|c|}
\hline & & $\begin{array}{c}\text { CCX } \\
\text { METODOLOGIA }\end{array}$ & $\begin{array}{l}\text { COBAS Integra } \\
\text { METODOLOGIF }\end{array}$ \\
\hline \multirow[t]{2}{*}{ Indices Lipídicos } & Categorias & & \\
\hline & $130-160 \mathrm{mg} / \mathrm{dl}$ & 28 & 28 \\
\hline \multirow[t]{3}{*}{ Não-HDL-c** } & $>160$ mg/dl & 56 & 64 \\
\hline & Subtotal & 84 & 92 \\
\hline & $\leq 2,0(200 / 100)$ & 21 & 50 \\
\hline \multirow[t]{3}{*}{ CT/LDL-c** } & $\leq 1,54(200 / 130)$ & - & 41 \\
\hline & Subtotal & 21 & 91 \\
\hline & $\geq 2,5$ (Homens) & 12 & 31 \\
\hline \multirow[t]{3}{*}{ LDL-c/HDLc*** } & $\geq 2,0$ (Mulheres) & 21 & 52 \\
\hline & Subtotal & 33 & 83 \\
\hline & $\geq 3,75$ (Homens) & 12 & 18 \\
\hline \multirow[t]{3}{*}{$\mathrm{TG} / \mathrm{HDL}-\mathrm{c}^{\#}$} & $\geq 3,0$ (mulheres) & 19 & 32 \\
\hline & Subtotal & 31 & 50 \\
\hline & Homens & 4 & 4 \\
\hline \multirow[t]{2}{*}{ Apo B/HDL-c"\#\# } & Mulheres & 13 & 15 \\
\hline & Subtotal & 17 & 19 \\
\hline \multicolumn{4}{|c|}{ 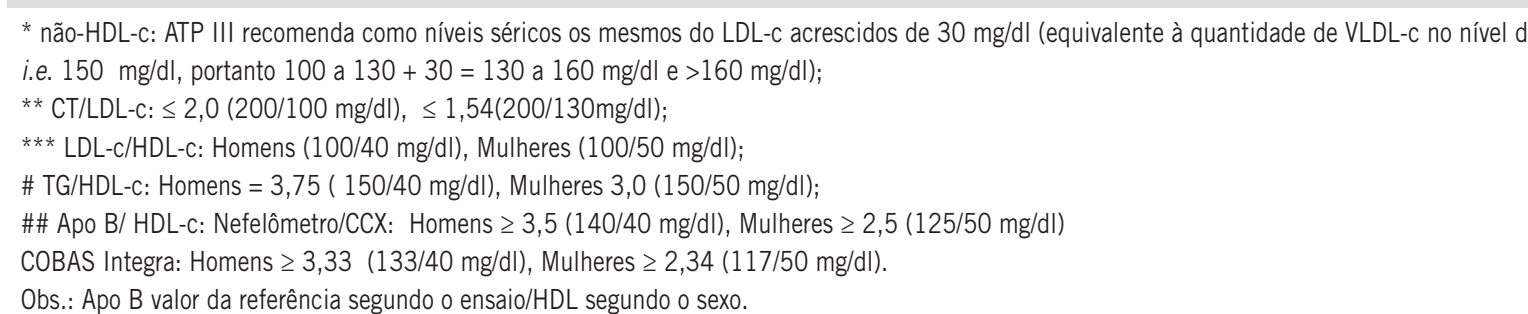 } \\
\hline
\end{tabular}


Níveis de não HDL-c nos grupos de pacientes com relação Apo B/HDL-c conforme

recomendado e com relação Apo B/HDL-c superior ao recomendado pelas III Diretrizes

Tabela 8

Brasileiras sobre Dislipidemias

Relação Apo B/HDL-c* Recomendado

$$
\text { Média } \pm \text { DP }
$$

Não HDL-c (CCX/

Nefelômetro)

$159,4 \pm 32,9$

$170,4 \pm 37,1$

Não HDL-C

(COBAS Integra
Relação Apo B/HDL-c Superior ao Recomendado

Média \pm DP

400)

*Apo B/ HDL-c: Nefelômetro/CCX : Homens $\geq 3,5$ (140/40 mg/dl), Mulheres $\geq 2,5$ (125/50 mg/dl)

COBAS Integra: Homens $\geq 3,33(133 / 40 \mathrm{mg} / \mathrm{dl})$, Mulheres $\geq 2,34(117 / 50 \mathrm{mg} / \mathrm{dl})$.

** $p<0.001$

Obs.: Apo B valor da referência segundo o ensaio/HDL segundo o sexo.

\section{Discussão}

As amostras biológicas analisadas são de pacientes com hipertensão. Deles, além de hipertensos, 20,2\% são diabéticos, todos diagnosticados e em terapia medicamentosa. No grupo analisado, 57,6\% apresentaram pressão arterial (PA) além do recomendado, embora estivessem sob intervenção medicamentosa anti-hipertensiva, necessitando de melhor monitoramento e/ou reavaliação da terapia.

A doença coronariana tem origem multifatorial e tradicionalmente o controle da PA, das dislipidemias e do diabetes constituía o indicador que avaliava a estimativa do risco individual(1). Entretanto, na prática, outros fatores de risco (FR) que avaliam o indivíduo de forma global devem ser considerados ${ }^{(4)}$. Os dados do Framingham Heart Study ilustram o efeito multiplicador dos vários FRs, notadamente a dislipidemia( ${ }^{(4)}$.

As III Diretrizes Brasileiras sobre Dislipidemias classificam o aumento sérico de CT como sendo de elevação moderada (limítrofe) entre 200 e $239 \mathrm{mg} / \mathrm{dl}$ e alta, superior a 240 $\mathrm{mg} / \mathrm{dl}$, respectivamente, como risco para desenvolvimento de doença coronariana ${ }^{(16)}$, ratificadas pelas IV Diretrizes ${ }^{(17)}$. No estudo realizado o número de pacientes classificados como de elevação sérica moderada e alta para o CT foi semelhante quando utilizamos metodologias e equipamentos de diferentes procedências. Perfil idêntico foi verificado para os parâmetros de TG, Apo A, e Lp(a) (Tabela 5), apesar de as médias obtidas nos parâmetros citados haverem apresentado diferença significativa quando observada a procedência dos ensaios (Tabela 6). Assim, considerando os parâmetros lipídicos citados, as intervenções hipolipemiantes aplicadas aos pacientes com níveis séricos superiores aos recomendados teriam procedimentos idênticos, independente da procedência dos ensaios, haja vista as classificações semeIhantes encontradas nos grupos, atendendo às expectativas e necessidades terapêuticas.

No entanto, o nível sérico elevado de LDL é um dos FRs mais importantes para o desenvolvimento da aterosclero$\mathrm{se}^{(2)}$, e sua elevação está associada a maior incidência de DAC ${ }^{(19)}$. Os níveis séricos de LDL-C são utilizados como indicadores primários das estratégias de redução de lipídios, sendo, contudo, importante considerar a variedade clínica de suas medidas para determinar o risco cardiovascular ${ }^{(26)}$ (Adult Treatment Panel III [ATP III]). A recomendação do ATP III é de redução do LDL-C para níveis séricos inferiores a $100 \mathrm{mg} / \mathrm{dl}$ em pacientes de alto risco(17). E estudos recomendam terapia mais agressiva, apontando a redução do LDL-C para níveis séricos inferiores a $70 \mathrm{mg} / \mathrm{dl}$ como eficaz para a diminuição de desfechos de $\operatorname{DAC}(3,11,17,20,24,27)$. Os níveis séricos de LDL-C determinados em pacientes hipertensos por meio de metodologias e equipamentos diferentes resultaram em número de pacientes classificados que apresentaram elevação intermediária (100-130 mg/dl) semelhante e alta elevação (> $130 \mathrm{mg} / \mathrm{dl}$ ) muito diferente, como podemos verificar na Tabela 5 . Em consequência dessa diferença caracterizada, a decisão a respeito da conduta terapêutica pode ser inadequada, dependendo das metodologias e dos equipamentos utilizados. Estudos cujas publicações datam desde 1998 demonstram que considerável variabilidade analítica de LDL-C pode ser verificada em decorrência da utilização de metodologias baseadas em princípios analíticos distintos $(5,9,8,28,31,33,38)$.

Alguns estudos preconizam a utilização de índices 
lipídicos como forma de aumentar a sensibilidade em predizer o risco cardiovascular determinado pelas dislipidemias $^{(30,34,36,37)}$. Em nosso estudo verificamos que os índices de CT/LDL-C, LDL-C/HDL-C e TG/HDL-C apresentaram diferenças em relação ao número de pacientes com valores não recomendados quando comparamos os resultados obtidos pela utilização de metodologias e equipamentos distintos (Tabela 7).

O índice lipídico obtido pela razão de ApoB/HDL-C esteve fortemente associado a aterosclerose em estudo de coorte em adultos sadios no National Health and Nutrition Examination Surveys e, segundo os autores, é uma medida de maior precisão para monitorar e determinar condutas terapêuticas hipolipemiantes ${ }^{(15)}$. $\mathrm{O}$ não-HDL-C foi um bom preditor de eventos cardiovasculares no estudo de Ridker et al. ${ }^{(30)}$. No estudo que realizamos, esses índices, quando obtidos nos ensaios de diferentes procedências, apresentaram semelhança quando analisado o número de pacientes com valores não recomendados (Tabela 7).

Os consensos permanecem com o LDL-C como alvo primário da terapia e o não-HDL-C como meta secundária a ser atingida ${ }^{(16)}$ (ATP III). Tal justificativa se deve ao fato de o LDL-C já estar incorporado à prática médica e de que, quando os níveis séricos de TG são inferiores a $200 \mathrm{mg} / \mathrm{dl}$, a correlação entre LDL-C e não-HDL-C é praticamente 1 (ATP III). No entanto, ao calcular-se o não-HDL-C estão sendo avaliadas as partículas potencialmente aterogênicas (LDL, lipoproteína de densidade intermediária [IDL] e remanescente de VLDL), além de excluir aquela presente na HDL e considerada protetora ${ }^{(10)}$, não observada na dosagem sérica isolada de LDL-C ${ }^{(10,26)}$. Por outro lado, Liu, et al. verificaram que em pacientes com concentrações séricas de TG menor que $200 \mathrm{mg} / \mathrm{dl}$, em relação a iguais ou maiores, o nãoHDL-C permanecia como forte preditor de risco para DCV ${ }^{(21)}$. Em nosso estudo as médias de TG encontradas foram de 171,8 mg/dl (CCX) e 189,8 mg/dl (COBAS Integra 400), inferiores a $200 \mathrm{mg} / \mathrm{dl}$, porém superiores ao referendado $(<150 \mathrm{mg} / \mathrm{dl}$ ) (III Diretrizes, ATP III), e o conhecimento do nível sérico do não-HDL-C avaliou as partículas potencialmente aterogênicas no grupo de hipertensos analisados, conforme estudo realizado por Liu et al. ${ }^{(21)}$.

A avaliação do perfil lipídico de forma criteriosa em pacientes hipertensos e/ou diabéticos torna-se imprescindível, tendo-se em vista o efeito sinérgico que isso poderá acarretar ${ }^{(4)}$, e as metodologias analíticas bem estabelecidas com controle de qualidade rígido estabelecido pelas sociedades existentes (Sociedade Brasileira de Análises Clínicas [SBAC], Sociedade Brasileira de Patologia Clínica [SBPC]), além de controles internos, devem ser seguidas. No entanto, no grupo de hipertensos analisados, verificou-se que, embora aplicadas as operações padrão de boas práticas para o laboratório clínico (BPLC), a utilização de metodologias de diferentes procedências apresentou resultados discordantes em um mesmo paciente, principalmente a LDL, o que implicaria ao clínico diferentes condutas hipolipemiantes. O estudo permite aferir, ainda, que as partículas aterogênicas do não-HDL-C, quando aplicado ao mesmo paciente, parecem ser evidenciadas, independente da metodologia e do equipamento utilizado.

Por outro lado, associar o não-HDL-C à relação Apo $\mathrm{B} / \mathrm{HDL}-\mathrm{C}$ poderia proporcionar critérios ainda mais rígidos em relação a condutas hipolipemiantes, pois, em nosso estudo, o grupo de hipertensos que apresentou a relação ApoB/HDL-C elevada, da mesma forma mostrou não-HDL-C superior ao recomendado, independente da procedência dos ensaios. E a média de não-HDL-C do grupo com Apo B/HDL-C elevado apresentou diferença estatisticamente significativa $(p<0,001)$ em relação aos que permaneceram em comparação com a relação Apo B/HDL-C desejável, independente da metodologia e do equipamento utilizados (Tabela 8).

Os resultados deste estudo permitem concluir que: a diferença caracterizada em relação ao LDL-C pode resultar em conduta terapêutica inadequada e que o não-HDL-C se caracterizou como importante fator de correção interensaios de parâmetros lipídicos e que sua associação à relação Apo B/HDL-C pode ser um fator adicional em relação à decisão quanto às condutas hipolipemiantes a serem adotadas. Entretanto, estudos com outras populações deverão ser realizados no sentido de referendar os resultados obtidos. 


\section{Referências}

1. ASSMANN, G. T. Lipid metabolism disorders and coronary heart disease: MMV. Medizin Verlag, 1993.

2. ATP II. Summary of the second report of the National Cholesterol Education Program (NCEP), Expert Panel on Detection, Evaluation and Treatment of High Blood Cholesterol in Adults (Adult Treatment Panel II). Circulation, v. 89, p. 1333-45, 1994.

3. CANNON, C. P. et al. Intensive versus moderate lipid lowering with statins after acute coronary syndromes. N Engl J Med, v. 350, p. 1495-504, 2004.

4. CASTELLI, W. P. et al. Chosleterol and lipids in the risk of coronary artery disease: The Framinghm Heart Study. Can J Cardiol, suppl. A, p. 5A-10A, 1988.

5. CORDOVA, C. M. M. et al. Avaliação da dosagem direta do colesterol-LDL em amostras de sangue de 10.664 pacientes em comparação com o uso da fórmula de Friedewald. Arq Bras Cardiol, v. 83, n. 6, p. 476-81, 2004.

6. DATASUS. Disponível em: <http://www.datasus.gov.br>. Acesso em: mar. 2007.

7. DAVIES, C. et al. A single cholesterol measurement underestimate the risk of CHD. An empirical example from the Lipid Research Clinics mortality follow-up study. JAMA, v. 264, p. 3044-6, 1990.

8. ESTEBAN-SALAN, M. et al. Analytical and clinical evaluation of two homogeneous assays for LDLcholesterol in hyperlipidemic patient. Clin Chem, v. 46, p. 1121-31, 2000.

9. FEl, H. et al. Evaluation of two different homogeneous assays for LDL-cholesterol in lipoprotein-X-positive serum. Clin Chem, v. 46, p. 1351-6, 2000.

10. FROST, P.H. et al. For the Systolic Hypertension in the Elderly Research Group. Serum lipids and incidence of coronary heart disease. Findings from the Systolic Hypertension in the Elderly Program. Circulation, v. 94, p. 2381-8, 1998.

11. GRUNDY, S. M. et al. Implications of recent clinical trials for the National Cholesterol Education Program Adult Treatment Panel III Guidelines. Circulation, v. 110, p. 227-39, 2004.

12. HALME, I. et al. Oslo Study: diet and antismoking advice: additional results from a 5-years primary preventative trial in middle-aged men. Prev Med, v. 14, p. 270-92, 1985.

13. HALPERIN, R. O. et al. Dyslipidemia and risk of incident hypertension in men. Hypertension, v. 47, p. 45-50, 2006.

14. HARMAN, D. Atherosclerosis: a hypothesis concerning the initiating steps in pathogenesis. J Gerontol, v. 12, p. 199-202, 1957.

15. HSIA, S. H. et al. A population-based, cross-sectional comparison of lipid-related indexes for symptoms of atherosclerosis disease. Am J Cardiol, v. 98, p. 1047-52, 2006.

16. III DIRETRIZES BRASILEIRAS SOBRE DISLIPIDEMIAS E DIRETRIZES DE PREVENÇÃO DA ATEROSCLEROSE DA SOCIEDADE BRASILEIRA DE CARDIOLOGIA. Arq Bras Cardiol, v. 77, supl. III, p. 1-48, 2001.
17. IV DIRETRIZES BRASILEIRAS SOBRE DISLIPIDEMIAS E DIRETRIZES DE PREVENÇÃO DA ATEROSCLEROSE DA SOCIEDADE BRASILEIRA DE CARDIOLOGIA. Arq Bras Cardiol, v. 88, supl. I, p. 1-19, 2007.

18. JEPPESEN, J. etal. Triglyceride concentration and ischemic heart disease. An eight-year follow-up in the Copenhagen Male Study. Circulation, v. 97, p. 1029-35, 1998.

19. LaROSA, JC et al. The cholesterol facts. A summary of the evidence relating dietary fats, serum cholesterol and coronary heart disease. A joint statement by the American Heart association and the National Heart, Lung and Blood Institute. The Task Force on Cholesterol Issues, American Heart Association. Circulation, v. 81, p. 1721-7, 1990.

20. LaROSA, J. C. et al. Treating to New Targets (TNT) Investigators. Intensive lipid lowering with atorvastatin in patients with stable coronary disease. N Eng/ J Med, v. 352, p. 1425-35, 2005.

21. LIU, J. et al. Non-hight-density lipoprotein and very-lowdensity lipoprotein cholesterol and their risk predictive values in coronary heart disease. The American Journal of Cardiology, v. 98, p. 1363-8, 2006.

22. MANNINEN, V. et al. Joint effects of serum triglyceride and LDL cholesterol and HDL cholesterol concentrations on coronary heart disease risk in the Helsinki Heart Study. Circulation, v. 85, p. 37-44, 1992.

23. MPFER, M. J. et al. A prospective study of triglyceride level, low-density lipoprotein particle diameter, and risk of myocardial infarction. JAMA, v. 276, p. 882-7, 1996.

24. MÜLLER, C. Xanthomata, hypercholesterolemia, angina pectoris. Acta Med Scand, v.89, p. 75-84, 1938.

25. NISSEN, S. et al. Effect of intensive compared with moderate lipid-lowering therapy on progression on coronary atherosclerosis. A randomized controlled trial. JAMA, v. 291, p. 1071-80, 2004.

26. NISSEN, S. E. et al. Effect of very hight-intensity statin therapy on regression of coronary atherosclerosis: The ASTEROID Trial. JAMA, v. 295, p. 1556-65, 2006.

27. OTVOS, J. D. et al. LDL particles, but not LDL cholesterol, are hightly elevated in the metabolic syndrome: results from. The Framingham Offspring Study. Circulation, v. 108, suppl. IV, p. 740-6, 2003.

28. PEDERSEN, T. R. et al. Incremental Decrease in End Points Through Aggressive Lipid Lowering (IDEAL) Study Group. High-dose atorvastatin vs. usual-dose sinvastatin for secondary prevention after myocardial infarction: the IDEAL study: a randomized controlled trial. JAMA, v. 294, p. 2437-45, 2005.

29. PIVA, J. P. J.; FERNANDES, T. R. L. Comparação analítica de valores de LDL-colesterol utilizando a dosagem direta e o cálculo pela fórmula de Friedewald. RBAC, v. 40, n. 4, p. 279-83, 2008.

30. REPORT OF THE NATIONAL CHOLESTEROL EDUCATION PROGRAM EXPERT PANEL ON DETECTION, EVALUATION AND TREATMENT OF HIGH BLOOD CHOLESTEROL IN ADULTS. THE EXPERT PANEL. Arch Intern Med, v. 148, p. 36-42, 1988. 
31. RIDKER, P. M. etal. Non-HDL cholesterol, apolipoproteins A-I and B100, standard lipid measures, lipid ratios and CRP as risk factors for cardiovascular disease in Women. JAMA, v. 294, p. 326-33, 2005.

32. RIFAI, N. et al. Analytical and clinical performance of a homogeneous enzymatic LDL-cholesterol assay compared with the ultracentrifugationdextran sulfate-Mg2+ method. Clin Chem, v. 44, p. 1242-50, 1998.

33. ROSINI, N. et al. Estudo de prevalência e multiplicidade de fatores de risco cardiovascular em hipertensos do município de Brusque, SC. Arquivos Brasileiros de Cardiologia, v. 86, p. 219-22, 2006.

34. SAHU, S.; CHAWLA, R.; UPPAL, B. Comparison of two methods of estimation of low density lipoprotein cholesterol, the direct versus Friedewald estimation. Indian Journal of Clinical Biochemistry, v. 20, n .2, p. 54-61, 2005.

35. SHAI, I.; et al. Multivariate assessment of lipid parameters as predictors of coronary heart disease among postmenopausal women. Potential implications for clinical guidelines. Circulation, v. 110, p. 2824-30, 2004.
36. SUMMARY OF THE SECOND REPORT OF THE NETIONAL CHOLESTEROL EDUCATION PROGRAM (NCEP), EXPERT PANEL ON DETECTION, EVALUATION, AND TREATMENT OF HIGH BLOOD CHOLESTEROL IN ADULTS (ATP II). JAMA, v. 269, p. 3015-21, 1993.

37. SUNDSTRÖM, J. et al. Left ventricular concentric remodeling rather than left ventricular hypertrophy is related to the insulin resistance syndrome in elderly men. Circulation, v. 101, p. 2595-600, 2000.

38. SUSKIN, N. et al. Glucose and insulin abnormalities relate to functional capacity in patients with congestive heart failure. Eur Heart J, v. 21, p. 1368-75, 2000.

39. SRIVASTAVA, M. et al. Variation in different lipoprotein fractions if estimated by different methodologies. Indian Heart J, v. 58, p. 239-44, 2006.

40. WARNICK, G. R. et al. Impact of the Third Cholesterol Report from the Adult Treatment Panel of the National Cholesterol Education Program on the Clinical Laboratory. Clinical Chemistry, v. 48, p. 11-7, 2002.

41. YUSUF, S. et al. Effect of potentially modifiable risk factors associated with muocardial infarction in 52 countries (The INTERHEART Study): case-control study. Lancet, v. 364, p. 937-952, 2004. 